\title{
Jensen measures, hyperconvexity and boundary behaviour of the pluricomplex Green function
}

\author{
by Magnus Carlehed (Östersund), Urban Cegrell (Umeå) and \\ FRANK WIKSTRÖM (Umeå)
}

\begin{abstract}
We characterise hyperconvexity in terms of Jensen measures with barycentre at a boundary point. We also give an explicit formula for the pluricomplex Green function in the Hartogs triangle. Finally, we study the behaviour of the pluricomplex Green function $g(z, w)$ as the pole $w$ tends to a boundary point.
\end{abstract}

1. Introduction. The pluricomplex Green function can be defined in analogy with the classical Green function for the Laplace operator as follows:

Definition 1.1. Let $\Omega$ be an open, connected subset of $\mathbb{C}^{N}$ and let $w \in \Omega$. Define

$$
g(z, w)=\sup \left\{u(z): u \in \mathcal{L}_{w}, u \leq 0\right\}
$$

where $\mathcal{L}_{w}=\{u \in \mathcal{P} \mathcal{S H}(\Omega): u(\zeta)-\log |\zeta-w| \leq O(1)$ as $\zeta \rightarrow w\}$. The function $g$ is called the pluricomplex Green function with pole at $w$. We sometimes write $g_{\Omega}$ if we want to emphasise the dependence on the domain.

We refer to the monograph by Klimek [14] for the basic properties of this function. It is well known that

$$
\lim _{\zeta \rightarrow z} g(\zeta, w)=0
$$

for every boundary point $z \in \partial \Omega$ if and only if $\Omega$ is hyperconvex.

The first part of our paper contains a characterisation of hyperconvexity in terms of Jensen measures and an explicit formula for the pluricomplex Green function in the Hartogs' triangle. As far as the authors know, this is the first known explicit example of the pluricomplex Green function in

1991 Mathematics Subject Classification: Primary 32F05; Secondary 32A07, 32F07, $32 \mathrm{H} 15$.

Key words and phrases: hyperconvexity, Jensen measures, Reinhardt domains, Hartogs' triangle, pluricomplex Green function. 
a pseudoconvex domain that is not hyperconvex. This part also gives a characterisation of hyperconvex Reinhardt domains.

The second part of the paper is a discussion of the behaviour of the pluricomplex Green function as the pole $w$ tends to a boundary point.

Throughout the paper, we will use $\Delta$ to denote the unit disc in $\mathbb{C}$ and $\mathcal{O}\left(E_{1}, E_{2}\right)$ to denote the family of all holomorphic mappings from $E_{1}$ to $E_{2}$.

The authors would like to thank the Department of Mathematics and Statistics, University of Canterbury, Christchurch for their kind hospitality during the authors' visit. We would also like to thank Professor Włodzimierz Zwonek for pointing out an error in a previous version.

\section{Jensen measures and hyperconvexity}

Definition 2.1. Let $\Omega$ be a bounded domain in $\mathbb{C}^{N}$, and let $\mu$ be a positive, regular Borel measure supported on $\bar{\Omega}$. We say that $\mu$ is a Jensen measure with barycentre $z$ if

$$
u(z) \leq \int_{\bar{\Omega}} u d \mu
$$

for every continuous function $u: \bar{\Omega} \rightarrow[-\infty, \infty)$ that is plurisubharmonic in $\Omega$. We denote by $\mathcal{J}_{z}$ the set of Jensen measures having barycentre $z$.

This is a slightly different definition of Jensen measures than the usual one, since we allow the measures to have support in $\bar{\Omega}$ and since we also consider Jensen measures for boundary points.

It is possible to estimate the maximal mass of Jensen measures as follows.

Proposition 2.2. Let $\Omega$ be a bounded domain in $\mathbb{C}^{N}$. Take $z, w \in \bar{\Omega}$, $z \neq w$. Then for every Jensen measure $\mu \in \mathcal{J}_{z}$, and every $0<r<|z-w|$,

$$
\mu(B(w, r)) \leq \frac{\log \frac{|z-w|}{\operatorname{diam}(\Omega)}}{\log \frac{r}{\operatorname{diam}(\Omega)}} .
$$

In particular, $\mu(B(w, r)) \rightarrow 0$ as $r \rightarrow 0$.

Proof. Let

$$
u(\zeta)=\log \frac{|\zeta-w|}{\operatorname{diam}(\Omega)}
$$

Then $u$ is a negative plurisubharmonic function on $\Omega$, continuous on $\bar{\Omega}$. 
Hence

$$
\begin{aligned}
\log \frac{|z-w|}{\operatorname{diam}(\Omega)} & =u(z) \leq \int_{\bar{\Omega}} u(\zeta) d \mu(\zeta) \\
& \leq \int_{B(w, r)} u(\zeta) d \mu(\zeta) \leq \log \left(\frac{r}{\operatorname{diam}(\Omega)}\right) \mu(B(w, r)) .
\end{aligned}
$$

Rearrangement gives us the desired result.

REMARK. The above estimate is sharp (up to a constant) if $w$ is an interior point of $\Omega$, as shown by the following example: Let $B$ denote the unit ball in $\mathbb{C}^{N}$ and take $r<1$. Consider the relative extremal function

$$
V(z)=\sup \left\{u(z): u \in \mathcal{P} \mathcal{S H}(B), u \leq-\chi_{B(0, r)}\right\} .
$$

It is well known [14] that

$$
V(z)=\max \left\{\frac{\log |z|}{-\log r},-1\right\} .
$$

For $|z|>r$, Edward's theorem [10] shows that $V(z)=-\sup \{\mu(B(0, r))$ : $\left.\mu \in \mathcal{J}_{z}\right\}$.

LEMMA 2.3. If $\left\{\mu_{j}\right\}_{j=1}^{\infty}$ is a sequence of positive measures such that $\mu_{j}$ converges weak-* to $\mu$ and if $\varphi$ is an upper semicontinuous function with compact support, then

$$
\varlimsup_{j \rightarrow \infty} \int \varphi d \mu_{j} \leq \int \varphi d \mu
$$

For a proof, see Lemma I.1 in Cegrell [5].

LEMma 2.4. Let $\Omega$ be a bounded domain in $\mathbb{C}^{N}$. Let $\left\{z_{n}\right\} \subset \bar{\Omega}$ be a sequence of points converging to $z$. For each $n$, let $\mu_{n} \in \mathcal{J}_{z_{n}}$. Then there is a subsequence $\mu_{n_{j}}$ and a measure $\mu \in \mathcal{J}_{z}$ such that $\mu_{n_{j}}$ converges weak-* to $\mu$.

Proof. First, using the Banach-Alaoglu theorem, we observe that $\bigcup_{z \in \bar{\Omega}} \mathcal{J}_{z}$ is contained in a weak-* compact set. Hence, by passing to a subsequence, we may assume that $\mu_{n}$ converges to some probability measure $\mu$ supported on $\bar{\Omega}$. In fact, $\mu$ is a Jensen measure for $z$, since if $u \in C(\bar{\Omega}) \cap \mathcal{P S H}(\Omega)$, then

$$
\int u d \mu=\lim _{n \rightarrow \infty} \int u d \mu_{n} \geq \lim _{n \rightarrow \infty} u\left(z_{n}\right)=u(z) .
$$

This shows that $\mu \in \mathcal{J}_{z}$.

Other definitions of Jensen measures allowing barycentres on $\partial \Omega$ are also possible; for example, one might consider Jensen measures for upper bounded plurisubharmonic functions, but this would give rise to additional complications. With this definition, it is not immediately clear whether Lemma 2.4 holds. 
The notion of hyperconvexity is an important concept in pluripotential theory. Hyperconvexity is defined in a way modelling the notion of regularity for the Dirichlet problem for the Laplace operator. More precisely:

Definition 2.5. Let $\Omega$ be a bounded domain in $\mathbb{C}^{N}$. We say that $\Omega$ is hyperconvex if there is a negative, continuous plurisubharmonic exhaustion function for $\Omega$.

In fact, for hyperconvexity, it is enough to have a bounded plurisubharmonic exhaustion function, or indeed a weak plurisubharmonic barrier for every boundary point. This fact is perhaps most clearly stated by Błocki [3]. For more details on these questions, see also Aytuna [1].

DeFinition 2.6. Let $\Omega \subset \mathbb{C}^{N}$ be a bounded domain, and let $z \in \partial \Omega$. We say that $u \in \mathcal{P} \mathcal{S H}(\Omega)$ is a weak plurisubharmonic barrier at $z$ if $u$ is a non-constant negative function such that

$$
\lim _{\zeta \rightarrow z} u(\zeta)=0 .
$$

THEOREM 2.7. Let $\Omega \subset \mathbb{C}^{N}$ be a bounded domain. Then $\Omega$ is hyperconvex if and only if every boundary point has a weak plurisubharmonic barrier.

Pr o of. See Błocki [3].

It is interesting to note that Jensen measures can be used to give a characterisation of hyperconvexity. Indeed, we have:

TheOREm 2.8. Let $\Omega$ be a bounded domain in $\mathbb{C}^{N}$. Then $\Omega$ is hyperconvex if and only if, for every $z \in \partial \Omega$ and every Jensen measure $\mu \in \mathcal{J}_{z}(\bar{\Omega}), \mu$ is supported on $\partial \Omega$.

Proof. Assume that $\Omega$ is hyperconvex. Let $u$ be a continuous, negative plurisubharmonic exhaustion function for $\Omega$. Take any boundary point $z \in$ $\partial \Omega$ and any $\mu \in \mathcal{J}_{z}$. Then

$$
0=u(z) \leq \int_{\bar{\Omega}} u d \mu .
$$

But, $u \leq 0$ on $\bar{\Omega}$ and $\mu$ is a positive measure, thus $u=0$ a.e. [ $\mu$ ]. Since $u<0$ in $\Omega$, this implies that $\mu(\Omega)=0$.

Conversely, take an open, relatively compact subset $E$ in $\Omega$ and consider the following construction (which is closely related to the relative extremal function for $E$ ):

$$
u(z)=\sup \{\varphi(z): \varphi \in C(\bar{\Omega}) \cap \mathcal{P S H}(\Omega), \varphi \leq 0, \varphi \leq-1 \text { on } E\} .
$$

Edward's theorem implies that

$$
u(z)=\inf \left\{-\int_{E} d \mu: \mu \in \mathcal{J}_{z}\right\}=-\sup \left\{\mu(E): \mu \in \mathcal{J}_{z}\right\} .
$$


Let $z \in \partial \Omega$ and assume that $\underline{\lim }_{\zeta \rightarrow z} u(\zeta)<0$. Take a sequence $z_{n} \rightarrow z$ such that

$$
u\left(z_{n}\right)<-\varepsilon \quad \text { for every } n .
$$

We can then find corresponding measures, $\mu_{n} \in \mathcal{J}_{z_{n}}$, such that $\mu_{n}(E)>\varepsilon$. By passing to a subsequence, using Lemma 2.4, we may assume that $\mu_{n}$ converge weak- ${ }^{*}$ to a probability measure $\mu \in \mathcal{J}_{z}$.

Then, using Lemma 2.3,

$$
\mu(\bar{E})=\int \chi_{\bar{E}} d \mu \geq \varlimsup_{n \rightarrow \infty} \int \chi_{\bar{E}} d \mu_{n}=\varlimsup_{n \rightarrow \infty} \mu_{n}(\bar{E}) \geq \varepsilon,
$$

which contradicts the assumption on Jensen measures for boundary points. This shows that $u^{*}$ is a bounded plurisubharmonic exhaustion function for $\Omega$. Hence $\Omega$ is hyperconvex.

Corollary 2.9. Let $\Omega$ be a bounded domain in $\mathbb{C}^{N}$ and let $z \in \partial \Omega$ and $w \in \Omega$. Then $\lim _{\zeta \rightarrow z} g(\zeta, w)=0$ if and only if every measure $\mu \in \mathcal{J}_{z}$ is supported on $\partial \Omega$.

Proof. The proof of Theorem 2.8 shows that there is a negative plurisubharmonic function $u$ on $\Omega$ tending to 0 at $z$ if and only if every Jensen measure with barycentre $z$ is supported on $\partial \Omega$. A standard construction (patching $u$ with a logarithmic pole) shows that the Green function tends to 0 at $z$. The converse is obvious.

Corollary 2.10. Let $\Omega$ be a bounded domain in $\mathbb{C}^{N}$ with $C^{1}$ boundary. If there is a boundary point $z$ and an analytic disc $\phi: \Delta \rightarrow \bar{\Omega}$ such that $\phi(0)=z$ and $\phi(\Delta) \not \subset \partial \Omega$, then $\Omega$ is not hyperconvex.

Proof. Let $\mu$ be the push-forward of the normalised Lebesgue measure on $\Delta$ by $\phi$. Then $\mu$ is a Jensen measure for $z$ which is not supported on $\partial \Omega$. To see that $\mu$ is indeed a Jensen measure, let $u$ be a plurisubharmonic function on $\Omega$, continuous up to the boundary. By a theorem of Fornæss and Wiegerinck [9], we can find a sequence $u_{j}$ of smooth functions, each plurisubharmonic on a neighbourhood of $\Omega$, such that $u_{j}$ converges to $u$ uniformly on $\bar{\Omega}$. Hence $u_{j}(z) \leq \int u_{j} d \mu$, since $u_{j} \circ \phi$ is subharmonic on (a neighbourhood of) $\Delta$, and by passing to a limit, we see that $\mu$ is a Jensen measure for continuous plurisubharmonic functions.

REMARK. At first sight it may seem that Corollary 2.10 follows immediately from the maximum principle for plurisubharmonic functions: Assume that $u$ is a bounded plurisubharmonic exhaustion function for $\Omega$, and take $\phi$ to be an analytic disc as in the statement of the corollary. Then $u \circ \phi$ is a negative subharmonic function on the unit disc in $\mathbb{C}$ such that $u \circ \phi$ vanishes for some interior point. The maximum principle would then force $u \circ \phi$ to vanish identically, contradicting the fact that $u$ is an exhaustion function for $\Omega$. The problem with this reasoning is that $u$ is only plurisubharmonic 
on $\Omega$, and the image of $\phi$ contains points of $\partial \Omega$. Hence we may only conclude that $u \circ \phi$ is subharmonic where it is negative, not on the whole of $\Delta$. Of course, if we know that $\phi^{-1}(\partial \Omega)$ is polar in $\Delta$, we may apply the maximum principle directly, and in this case it is not necessary to require any boundary regularity of $\Omega$.

EXAMPLE 2.11. The converse of Corollary 2.10 is not true as seen from the following example. Let $\alpha$ be a positive irrational number and define

$$
\Omega=\left\{\left(z_{1}, z_{2}\right) \in \mathbb{C}^{2}:\left|z_{1}\right|^{\alpha}<\left|z_{2}\right|<2\left|z_{1}\right|^{\alpha}\right\} \cap \Delta^{2} .
$$

It is not difficult to verify that $\Omega$ is pseudoconvex and that every boundary point of $\Omega$ except 0 admits a weak plurisubharmonic barrier. However, $0 \in$ $\partial \Omega$, and thus $\Omega$ is not hyperconvex, and there is no non-constant analytic disc $f: \Delta \rightarrow \bar{\Omega}$ such that $f(0)=0$. Seeking a contradiction, assume that there exists such a disc, $f(\zeta)=\left(f_{1}(\zeta), f_{2}(\zeta)\right)$, where $f_{1}(0)=f_{2}(0)=0$. Then either one of the components $f_{j}$ vanishes identically, which forces the other component to vanish as well, or we can write

$$
f_{j}(\zeta)=\zeta^{k_{j}} g_{j}(\zeta), \quad j=1,2,
$$

where $k_{j}$ are positive integers, and $g_{j}$ are holomorphic on $\Delta$ and $g_{j}(0) \neq 0$. By assumption, the image of $f$ is contained in $\bar{\Omega}$, hence

$$
|\zeta|^{k_{1} \alpha}\left|g_{1}(\zeta)\right|^{\alpha} \leq|\zeta|^{k_{2}}\left|g_{2}(\zeta)\right|^{k_{2}} \leq 2|\zeta|^{k_{1} \alpha}\left|g_{1}(\zeta)\right|^{\alpha}
$$

for every $\zeta \in \Delta$. Looking at the first inequality and letting $\zeta \rightarrow 0$ we deduce that $k_{1} \alpha \geq k_{2}$. Similarly, the second inequality implies that $k_{2} \geq k_{1} \alpha$. Hence $k_{1} \alpha=k_{2}$, which contradicts the assumption that $\alpha$ is irrational.

EXAmPLE 2.12. Let $T$ denote the Hartogs triangle, $T=\left\{\left(z_{1}, z_{2}\right) \in \mathbb{C}^{2}\right.$ : $\left.\left|z_{1}\right|<\left|z_{2}\right|<1\right\}$. Then $T$ is pseudoconvex, but not hyperconvex, since $\phi(\zeta)=$ $(0, \zeta)$ is an analytic disc passing through $(0,0) \in \partial T$ but not lying entirely in $\partial T$. This shows that the pluricomplex Green function for $T$ does not tend to 0 at the origin. On the other hand, one can show that the Jensen measures of all other boundary points are supported on $\partial T$. Hence, the Green function will tend to 0 at every boundary point of $\partial T$ except the origin.

In fact, it is possible to explicitly calculate the pluricomplex Green function for $T$. Let $\Delta^{2}$ denote the (unit) bidisc in $\mathbb{C}^{2}$, and define $E=\left\{\left(z_{1}, 0\right)\right.$ : $\left.\left|z_{1}\right|<1\right\}$. Let $f: \Delta^{2} \backslash E \rightarrow T$ be defined by $f\left(z_{1}, z_{2}\right)=\left(z_{1} z_{2}, z_{2}\right)$. Then $f$ is a biholomorphism. Let

$$
\begin{aligned}
g(z, w) & =g_{\Delta^{2}}\left(f^{-1}(z), f^{-1}(w)\right) \\
& =\log \max \left\{\left|\frac{z_{1} / z_{2}-w_{1} / w_{2}}{1-z_{1} \bar{w}_{1} /\left(z_{2} \bar{w}_{2}\right)}\right|,\left|\frac{z_{2}-w_{2}}{1-z_{2} \bar{w}_{2}}\right|\right\} .
\end{aligned}
$$

We claim that $g$ is the pluricomplex Green function for $T$. The set $E$ is pluripolar, hence the pluricomplex Green function for $\Delta^{2} \backslash E$ is the restriction 
of the Green function for $\Delta^{2}$ to $\Delta^{2} \backslash E$. However, the pluricomplex Green function is invariant under biholomorphisms.

Here, we see directly that for a fixed $w, g(z, w)$ will tend to 0 as $z$ tends to any boundary point except the origin. But $g(\varepsilon z, w)$ will have different limits for different $z$ as $\varepsilon \rightarrow 0$.

The above reasoning can also be used to compute the automorphism group for $T$. Let $\phi \in \operatorname{Aut}(T)$. Then $f^{-1} \circ \phi \circ f$ is an automorphism of $\Delta^{2} \backslash E$, hence an automorphism of $\Delta^{2}$ preserving $E$. It is easy to verify that an automorphism of $\Delta^{2}$ fixing $E$ must be a rotation in the $z_{2}$ variable and an arbitrary Möbius transformation in the $z_{1}$ variable. This means that

$$
f^{-1} \circ \phi \circ f(z)=\left(e^{i \theta} \frac{z_{1}-\alpha}{1-\bar{\alpha} z_{1}}, e^{i \psi} z_{2}\right),
$$

for some $\theta, \psi \in \mathbb{R}, \alpha \in \Delta$. Hence,

$$
\phi(z)=\left(e^{i(\theta+\psi)} \frac{z_{1} z_{2}-\alpha z_{2}^{2}}{z_{2}-\bar{\alpha} z_{1}}, e^{i \psi} z_{2}\right) .
$$

It is also possible to give a complete characterisation of hyperconvex Reinhardt domains. We recall that a domain $\Omega \subset \mathbb{C}^{N}$ is said to be Reinhardt if

$$
\left(z_{1}, \ldots, z_{N}\right) \in \Omega \Rightarrow\left(e^{i \theta_{1}} z_{1}, \ldots, e^{i \theta_{n}} z_{N}\right) \in \Omega,
$$

for every real $\theta_{1}, \ldots, \theta_{N}$. For completeness, we begin by the following proposition.

Proposition 2.13. Let $\Omega$ be a bounded pseudoconvex Reinhardt domain. Then the logarithmic image of $\Omega$ is convex.

P r o of. Assume that the logarithmic image $\widetilde{\Omega}$ of $\Omega$ is not convex. Then we can find a sequence of parallel line segments $L_{\tau} \subset \widetilde{\Omega}, 0 \leq \tau \leq 1$, where $\bigcup \partial L_{\tau} \Subset \widetilde{\Omega}$, but $\bigcup L_{\tau}$ is not relatively compact in $\widetilde{\Omega}$. Parametrise $L_{\tau}=P_{\tau}+a t,-\varepsilon \leq t \leq \varepsilon$. We may assume that all components of $a$ are integers (an arbitrarily small perturbation will ensure this). Then the pre-image of $L_{\tau}$ under the logarithmic map will be

$$
D_{\tau}=\left\{\left(z_{1}, \ldots, z_{N}\right) \in \mathbb{C}^{N}:\left|z_{j}\right|=C_{\tau, j} t^{a_{j}}, e^{-\varepsilon} \leq t \leq e^{\varepsilon}\right\} .
$$

Let $A$ denote the annulus $\left\{e^{-\varepsilon} \leq|\zeta| \leq e^{\varepsilon}\right\} \subset \mathbb{C}$, and define $d_{\tau}: A \rightarrow D_{\tau} \subset$ $\Omega$ by

$$
d_{\tau}(\zeta)=\left(C_{\tau, 1} \zeta^{a_{1}}, \ldots, C_{\tau, N} \zeta^{a_{N}}\right)
$$

Then $\left\{d_{\tau}\right\}$ is a family of "analytic annuli" such that $\bigcup \partial d_{\tau} \Subset \Omega$, but $\bigcup d_{\tau}$ is not relatively compact in $\Omega$. This contradicts the assumption that $\Omega$ is pseudoconvex. (This is a minor modification of the standard Kontinuitätsprinzip, see e.g. Krantz [15].) 
Theorem 2.14. A bounded pseudoconvex Reinhardt domain, $\Omega$, in $\mathbb{C}^{N}$ is hyperconvex if and only if $0 \notin \partial \Omega$ and for every point $z=\left(z_{1}, \ldots, z_{N}\right) \in \partial \Omega$ with some $z_{j}=0$, we have

$$
|\Omega| \cap \Pi=\emptyset
$$

for some plane $\mathbb{R}^{N} \supset \Pi=\left\{x \in \mathbb{R}^{N}: a \bullet(x-|z|)=0\right\}$ where $a_{j}=0$ for every $j$ such that $z_{j}=0$. Here $|\Omega|$ and $|z|$ denote the images of $\Omega$ and $z$, respectively, under the mapping

$$
\mathbb{C}^{N} \ni\left(\zeta_{1}, \ldots, \zeta_{N}\right) \mapsto\left(\left|\zeta_{1}\right|, \ldots,\left|\zeta_{N}\right|\right) \in \mathbb{R}^{N} .
$$

Proof. Assume that $0 \notin \partial \Omega$. Let $\widetilde{\Omega} \subset \mathbb{R}^{N}$ denote the logarithmic image of $\Omega$, i.e.

$$
\widetilde{\Omega}=\left\{\left(\log \left|z_{1}\right|, \ldots, \log \left|z_{N}\right|\right):\left(z_{1}, \ldots, z_{N}\right) \in \Omega\right\} .
$$

By assumption, $\Omega$ is pseudoconvex, hence $\widetilde{\Omega}$ is convex. Take any $z \in \partial \Omega$. It is enough to find a non-constant plurisubharmonic function $u$ on $\Omega$ such that $u(z) \geq u(\zeta)$ for all $\zeta \in \Omega$. Let $\widetilde{z} \in \partial \widetilde{\Omega}$ be the image of $z$ under the logarithmic map. First assume that $z_{j} \neq 0$ for every $1 \leq j \leq N$. By convexity, we can find a linear functional $L$ on $\mathbb{R}^{N}$ such that $\infty>L(\widetilde{z}) \geq L(x)$ for every $x \in \widetilde{\Omega}$, where $L(t)=\sum a_{j} t_{j}$. Define

$$
u(\zeta)=\sum_{j=1}^{N} a_{j} \log \left|\zeta_{j}\right| .
$$

Note that $u$ is plurisubharmonic outside the coordinate axes in $\mathbb{C}^{N}$ and that $u(\zeta)=L(\widetilde{\zeta})$. Hence $u(z) \geq u(\zeta)$ for every $\zeta \in \Omega$. In particular, $u$ is bounded above and plurisubharmonic on $\Omega$ outside the coordinate axes, and can be extended to a plurisubharmonic function on $\Omega$ taking its maximum in $z$. In the case where some of the coordinates of $z$ are zero, the above construction works, as the assumptions in the theorem allow us to take the corresponding $a_{j}$ 's to be zero. (Geometrically, there is a supporting hyperplane for $\widetilde{\Omega}$ which is parallel to the critical coordinate axes. This means that even though some coordinate of $\widetilde{z}$ is $-\infty$, the functional $L$ will have a finite value in $\widetilde{z}$.)

Conversely, assume that $0 \in \partial \Omega$. Fix a point $P$ in $\widetilde{\Omega}$ with rational coordinates. Choose a sequence of points $Q_{j} \in \widetilde{\Omega}$ such that $Q_{j} \rightarrow(-\infty, \ldots,-\infty)$ and each $Q_{j}$ has rational coordinates. By convexity of $\widetilde{\Omega}$, the line segment $L_{j}$ connecting $Q_{j}$ and $P$ is contained in $\widetilde{\Omega}$. As in the proof of Proposition 2.13 , for every $j$, we can find an analytic annulus $d_{j}: A_{j} \rightarrow \Omega$ in the pre-image of $L_{j}$ under the logarithmic map. After a suitable change of coordinates, we may assume that $A_{j}=\left\{\varrho_{j}<|\zeta|<1\right\}$. Since $Q_{j}$ converges to $(-\infty, \ldots,-\infty)$, we may arrange so that $\varrho_{j} \rightarrow 0$ as $n \rightarrow \infty$.

Now, let $\zeta_{j}=\sqrt{\varrho_{j}}$ and let $\omega_{j}$ denote the harmonic measure on $A_{j}$ with respect to $\zeta_{j}$. An easy computation shows that $\omega_{j}(\{|\zeta|=1\})=1 / 2$. Also 
note that $z_{j}=d_{j}\left(\zeta_{j}\right) \rightarrow 0$ as $j \rightarrow \infty$. Let $\mu_{j}=\left(d_{j}\right)_{*} \omega_{j}$ (the push-forward of $\omega_{j}$ under $d_{j}$ ). Then $\mu_{j}$ is a Jensen measure with barycentre $z_{j}$ with the property that $\mu_{j}\left(P^{\prime}\right)=1 / 2$ where $P^{\prime}$ is the pre-image of $P$ under the logarithmic map. Finally, with the help of Lemma 2.4, we may take a subsequence of $\left\{\mu_{j}\right\}$ converging weak-* to a Jensen measure for 0 . This measure is not supported on $\partial \Omega$, and hence, by Theorem $2.8, \Omega$ is not hyperconvex.

Similarly, if $z \in \partial \Omega$, some coordinates of $z$ are zero, say $z_{j}=0$ for $j \in J \subset\{1, \ldots, N\}$, and (2.1) is not satisfied, then the analytic disc

$$
f(\zeta)=\left(f_{1}(\zeta), \ldots, f_{N}(\zeta)\right)
$$

where $f_{j}(\zeta)=z_{j}$ if $j \notin J$ and $f_{j}(\zeta)=\varepsilon \zeta$ if $j \in J$, is a punctured analytic disc as in Corollary 2.10 (since only $f(0) \in \partial \Omega$, the assumption of $C^{1}$ boundary in the corollary is not necessary) provided that $\varepsilon>0$ is sufficiently small. (This last assertion also requires that $\widetilde{\Omega}$ is convex.)

REMARK. It has come to our attention that the problem of characterising hyperconvex Reinhardt domains has also been studied by Zwonek [24] using a different method.

\section{The Lempert function}

Definition 3.1. Let $\Omega$ be bounded domain in $\mathbb{C}^{N}$. Define the Lempert function $\delta: \Omega \times \Omega \rightarrow \mathbb{R}$ by

$$
\delta(z, w)=\inf \{\log |t|: \exists f \in \mathcal{O}(\Delta, \Omega) \text { such that } f(0)=z, f(t)=w\} .
$$

We recall that the Lempert function is the starting point for the definition of the Kobayashi distance. Lempert proved [18, 19] that for convex domains, $\delta(z, w)=g(z, w)$.

It is always the case that $g(z, w) \leq \delta(z, w)$ : Consider any analytic disc $\phi$ : $\Delta \rightarrow \Omega$, where $\phi(0)=z, \phi(t)=w$. Then, since $g(z, w)$ is plurisubharmonic in $z$, the function $u(\zeta)=g(\phi(\zeta), w)$ is subharmonic on $\Delta$. It is easy to verify that $u$ is negative on $\Delta$ and has a logarithmic pole at $\zeta=t$. Hence $u$ is in the defining family for the Green function on $\Delta$ and, thus,

$$
u(\zeta) \leq \log \left|\frac{t-\zeta}{1-\bar{t} \zeta}\right| .
$$

Hence $g(z, w)=u(0) \leq \log |t|$. Taking the infimum over all such discs, we obtain $g(z, w) \leq \delta(z, w)$.

Definition 3.2. Let $\Omega$ be a domain in $\mathbb{C}^{N}$. Following Wu [22], we say that $\Omega$ is taut if for any sequence $f_{n} \in \mathcal{O}(\Delta, \Omega)$ there exists a subsequence $f_{n_{j}}$ such that either

- $f_{n_{j}} \rightarrow f \in \mathcal{O}(\Delta, \Omega)$ locally uniformly, or 
- for each compact subset $K$ of $\Delta$ and each compact subset $L$ of $\Omega$, $f_{n_{j}}(K) \cap L$ is empty for all sufficiently large $j$.

The Kontinuitätsprinzip (see e.g. Krantz [15]) shows that every taut domain is pseudoconvex. Conversely, Kerzman and Rosay [13] showed that every pseudoconvex domain with $C^{1}$ boundary, and more generally, every hyperconvex domain, is taut. However, hyperconvexity is a stronger condition than tautness, as shown by the Hartogs triangle.

It is well known (cf. Jarnicki-Pflug [12]) that if $\Omega$ is a taut domain, then the Lempert function is continuous on $\Omega \times \Omega$ off the diagonal. This result can be somewhat improved.

THEOREM 3.3. Let $\Omega$ be a bounded taut domain such that $\partial \Omega$ contains no non-constant analytic disc. Then $\delta$ is continuous on $\bar{\Omega} \times \bar{\Omega} \backslash\{(z, z): z \in \bar{\Omega}\}$.

P r o of. Because of the symmetry of the Lempert function and the continuity on $\Omega \times \Omega$, we only need to consider $\bar{\Omega} \times \partial \Omega$. Let $\left(\zeta_{j}, \eta_{j}\right)$ be a sequence of points tending to $(z, w) \in \bar{\Omega} \times \partial \Omega$. Assume that

$$
\varliminf_{j \rightarrow \infty} \delta\left(\zeta_{j}, \eta_{j}\right)<0 .
$$

Then (after passing to a subsequence) there is an $\varepsilon>0$ such that $\delta\left(\zeta_{j}, \eta_{j}\right)<$ $-2 \varepsilon$ and corresponding analytic discs $f_{j}$ such that $f_{j}(0)=\zeta_{j}, f_{j}\left(t_{j}\right)=\eta_{j}$ and $\log \left|t_{j}\right|<-\varepsilon$. But, since $\Omega$ is taut, either there is a subsequence of the discs, $\left\{f_{j_{k}}\right\}$, converging locally uniformly to an analytic disc $f$, or the $f_{j}$ 's "diverge".

In the first case, we may also assume that $t_{j_{k}}$ converges to some $t$, where $|t| \leq e^{-\varepsilon}$. Using equicontinuity of the family $\left\{f_{j_{k}}\right\}$, we see that $f(t)=w$, $f(0)=z$, but since $w \in \partial \Omega$ and $f \in \mathcal{O}(\Delta, \Omega)$, this is a contradiction.

In the second case, by using Montel's theorem on every component of $\left\{f_{j}\right\}$, we see that a subsequence converges to a disc $f$, where $f(0)=z$, $f(t)=w$ for some $t$, and $f \in \mathcal{O}(\Delta, \bar{\Omega})$. Hence $f(\Delta) \subset \partial \Omega$, which contradicts the assumption on $\Omega$. Thus if we extend $\delta$ to 0 on $\partial \Omega \times \bar{\Omega} \cup \bar{\Omega} \times \partial \Omega$, we find that $\delta$ is continuous on $\bar{\Omega} \times \bar{\Omega} \backslash\{(z, z): z \in \bar{\Omega}\}$.

REMARK. Let $\Omega$ be a bounded taut domain and let $w \in \partial \Omega$. Define $\mathcal{D}_{w}$ as the union of (the images of) all analytic discs lying in $\partial \Omega$ and passing through $w$. Note that the proof of Theorem 3.3 shows that $\delta\left(\zeta_{j}, \eta_{j}\right) \rightarrow 0$ when $\zeta_{j} \rightarrow z$ and $\eta_{j} \rightarrow w$ as long as $z \notin \mathcal{D}_{w}$.

4. Boundary behaviour of the pluricomplex Green function. Let $\Omega$ be a bounded domain in $\mathbb{C}^{N}$. We now study the following question: Given a point $z \in \Omega$ and a point $w_{0} \in \partial \Omega$, is it true that

$$
\lim _{n \rightarrow \infty} g\left(z, w_{n}\right)=0
$$


for every sequence $w_{n}$ tending to $w_{0}$ ? The corresponding question for the classical Green function, $G$, is of no interest, since $G$ is symmetric, that is, $G(x, y)=G(y, x)$ for every $x$ and $y$. It is obvious that the answer to our question is "yes" if $\Omega$ is a hyperconvex domain for which the pluricomplex Green function is symmetric, for example, a convex domain.

Definition 4.1. Let $\Omega$ be a bounded domain in $\mathbb{C}^{N}$. We say that a point $w_{0} \in \partial \Omega$ has Property $\left(\mathrm{P}_{0}\right)$ if

$$
\lim _{w \rightarrow w_{0}} g(z, w)=0
$$

for every $z \in \Omega$. If the convergence is uniform in $z$ on compact subsets of $\bar{\Omega} \backslash\left\{w_{0}\right\}$, we say that $w_{0}$ has Property (P).

Remark. Property (P) was introduced by Coman [6].

ExAmple 4.2. Property $\left(\mathrm{P}_{0}\right)$ does not imply Property $(\mathrm{P})$. Take $\Omega=$ $\Delta^{2}$, the unit bidisc. Clearly, since $\Omega$ is convex, every boundary point has Property $\left(\mathrm{P}_{0}\right)$. However, not every boundary point has Property $(\mathrm{P})$. Take $w_{0}=(a, 1) \in \partial \Omega, 0<|a|<1$, and let $w_{n}=(a, 1-1 / n), z_{n}=(0,1-1 / n)$. Then $\left(z_{n}, w_{n}\right) \rightarrow\left((0,1), w_{0}\right)$, but $g\left(z_{n}, w_{n}\right)=\log |a| \neq 0$ for every $n$. Hence $w_{0}$ does not have Property $(\mathrm{P})$.

Definition 4.3. Let $\Omega$ be a domain in $\mathbb{C}^{N}$. Define

$$
c(z, w)=\sup \{\log |f(z)|: f \in \mathcal{O}(\Omega, \Delta), f(w)=0\} .
$$

The function $\tanh ^{-1} \exp c$ is called the Carathéodory pseudodistance on $\Omega$.

REMARK. It is easy to see that $c \leq g$.

A similar construction was proposed by Hervé [11] in connection with questions in infinite dimensions.

Definition 4.4. Let $\Omega$ be an open set in $\mathbb{C}^{N}$. Define

$$
e(z, w)=\sup \left\{g_{f(\Omega)}(f(z), f(w))\right\},
$$

where the supremum is taken over all non-constant holomorphic functions $f: \Omega \rightarrow \mathbb{C}$.

Remark. Note that $c \leq e \leq g$. The left-hand inequality follows from restricting the supremum in the definition of $e$ to functions from $\Omega$ into $\Delta$, and, in this case, we have $g_{f(\Omega)} \geq g_{\Delta}$. The right-hand inequality follows from the fact that $g$ is decreasing under holomorphic mappings [14].

Theorem 4.5. Let $\Omega$ be a domain in $\mathbb{C}^{N}$ and assume that $w_{0} \in \partial \Omega$ is a peak point for $A(\bar{\Omega})$. Then $w_{0}$ has property $(\mathrm{P})$. 
Proof. Let $f$ be a peak function for $w_{0}$, i.e. $f \in A(\bar{\Omega}), f\left(w_{0}\right)=1$ and $|f|<1$ on $\bar{\Omega} \backslash\left\{w_{0}\right\}$. Define

$$
v(z, w)=\log \left|\frac{f(z)-f(w)}{1-f(z) \overline{f(w)}}\right| .
$$

Hence $g(z, w) \geq c(z, w) \geq v(z, w)$ and from (4.1), it is clear that

$$
\lim _{w \rightarrow w_{0}} v(z, w)=0
$$

the convergence being uniform in $z$ on compact subsets of $\bar{\Omega} \backslash\left\{w_{0}\right\}$.

REMARK. The above proof actually shows that if there is a weak peak function for $w_{0} \in \partial \Omega$, that is, a non-constant holomorphic function $f \in$ $A(\bar{\Omega})$ such that $f\left(w_{0}\right)=1,|f| \leq 1$ on $\bar{\Omega}$, then $w_{0}$ has Property $\left(\mathrm{P}_{0}\right)$. In fact, we can even conclude that $g(z, w) \rightarrow 0$ uniformly in $z$ on compact subsets of $\bar{\Omega} \backslash f^{-1}(1)$. In a polydisc, this last conclusion is sharp, in the sense that we do not have locally uniform convergence on any larger set.

In particular, if $\Omega \subset \mathbb{C}^{2}$ has $C^{\omega}$ boundary, or more generally, if $\Omega \subset \mathbb{C}^{2}$ has smooth boundary of finite type [2], then every boundary point of $\Omega$ is a peak point for $A(\bar{\Omega})$. For smoothly bounded domains in higher dimensions, there are results on the existence of peak functions due to $\mathrm{Yu}$ [23] and Diederich and Herbort [7].

For strictly pseudoconvex domains with $C^{2}$ boundary, Carlehed [4] gave the following estimate on the speed of convergence:

$$
-g(z, w) \leq C \frac{\operatorname{dist}(z, \partial \Omega) \operatorname{dist}(w, \partial \Omega)}{|z-w|^{4}},
$$

where $C$ is some positive constant, depending on $\Omega$.

Proposition 4.6. Let $\Omega \subset \mathbb{C}^{N}$ be a bounded domain, and let $w_{0} \in \partial \Omega$. Assume that there exists a holomorphic function $f: \Omega \rightarrow \mathbb{C}$, continuous on $\bar{\Omega}$, such that $f\left(w_{0}\right)$ is a regular boundary point of $f(\Omega)$. Then $w_{0}$ has Property $\left(\mathrm{P}_{0}\right)$.

Proof. The assumptions imply that $e(z, w) \rightarrow 0$ as $w \rightarrow w_{0}$.

REMARK. A domain $\Omega$ is said to be $c$-finitely compact if every $c$-ball is relatively compact in $\Omega$. This property implies that all boundary points have Property $\left(\mathrm{P}_{0}\right)$. Examples of such domains are bounded pseudoconvex Reinhardt domains containing 0. (See [12] for details.)

Using the results of Poletsky [20, 21], Edigarian [8] and Lárusson and Sigurdsson [16], we can write the pluricomplex Green function as 


$$
\begin{aligned}
g(z, w) & =\sup \{u(z): u \in \mathcal{P S H}(\Omega), u(\cdot) \leq \delta(\cdot, w)\} \\
& =\inf \left\{\int_{\bar{\Omega}} \delta(\zeta, w) d \mu(\zeta): \mu \in \mathcal{M}_{z}\right\},
\end{aligned}
$$

where $\mathcal{M}_{z}$ denotes the set of all measures which are push-forward measures of the (normalised) Lebesgue measure on $\partial \Delta$ by functions in $\mathcal{A}_{z}=\{f \in$ $\mathcal{O}(\Delta, \Omega): f(0)=z\}$. Note that $\mathcal{M}_{z} \subset \mathcal{J}_{z}$.

It is interesting to note that for any $r>0$ and any $n$ such that $\left|w-w_{0}\right|$ $<r$, we have

$$
\begin{aligned}
g(z, w) & =\sup \left\{u(z): u \in \mathcal{P S H}(\Omega), u(\cdot) \leq \chi_{B\left(w_{0}, r\right)} \delta(\cdot, w)\right\} \\
& =\inf \left\{\int_{\bar{\Omega} \cap B\left(w_{0}, r\right)} \delta(\zeta, w) d \mu(\zeta): \mu \in \mathcal{M}_{z}\right\} .
\end{aligned}
$$

The second equality follows from using Poletsky's results on the function $\chi_{B\left(w_{0}, r\right)} \delta(\cdot, w)$. A priori, this common expression is greater than or equal to $g$, but it is in fact a member of the defining family for $g$, which establishes the first equality. This reasoning implies that $w_{0}$ having Property $\left(\mathrm{P}_{0}\right)$ only depends on the behaviour of the Lempert function close to $w_{0}$.

It also follows that if $\mu_{k}$ is a sequence in $\mathcal{M}_{z}$ such that

$$
\int_{\bar{\Omega} \cap B(w, r)} \delta(\zeta, w) d \mu_{k}(\zeta) \rightarrow g(z, w)
$$

then $\mu_{k}\left(\bar{\Omega} \backslash B\left(w_{0}, r\right)\right) \rightarrow 0$ as $k \rightarrow \infty$. Hence, "in the limit", the measure minimising the integral is supported on $\partial \Omega \cup B\left(w_{0}, r\right)$.

Remark. Poletsky's machinery requires, in the general case, that the infima are taken over push-forwards of the Lebesgue measure on the circle by analytic discs which extend to a neighbourhood of $\Delta$. In the construction of the Green function, it makes no difference to consider infima over larger classes of Jensen measures, as long as they are Jensen measures for the Green function. The reason is that if $\mu$ is such a measure, then

$$
\int \delta(\zeta, w) d \mu(\zeta) \geq \int g(\zeta, w) d \mu(\zeta) \geq g(z, w) .
$$

Here, the first inequality follows from $\delta \geq g$, and the second from $\mu$ being a Jensen measure for $g$ with barycentre $z$. In other words, an infimum as in (4.2) can never be smaller than $g$, even if we take the infimum over all Jensen measures with respect to $z$. In fact, if the class of measures we are considering contains the push-forwards of analytically extendible discs, the infimum will equal the Green function, because of Poletsky's results.

It is not known whether every boundary point in a hyperconvex domain has Property $\left(\mathrm{P}_{0}\right)$. We can, however, prove the following weaker result. 
TheOREM 4.7. Let $\Omega$ be a bounded, hyperconvex domain in $\mathbb{C}^{N}$, and let $\left\{w_{n}\right\}$ be a sequence in $\Omega$ tending to $w_{0} \in \partial \Omega$. Then there exists a pluripolar set $E \subset \Omega$ (dependent of the sequence) such that

$$
\varlimsup_{n \rightarrow \infty} g\left(z, w_{n}\right)=0 \quad \text { for every } z \in \Omega \backslash E .
$$

Proof. Let $h$ be a continuous exhaustion function for $\Omega$. Define

$$
u_{j}(z)=\sup _{n \geq j} g\left(z, w_{n}\right) .
$$

Then each $u_{j}^{*}$ is a bounded plurisubharmonic function, tending to 0 at $\partial \Omega$. Hence

$$
\begin{aligned}
\int_{\Omega}(-h)\left(d d^{\mathrm{c}} u_{j}^{*}\right)^{n} & =\int_{\Omega}\left(-u_{j}^{*}\right) d d^{\mathrm{c}} h \wedge\left(d d^{\mathrm{c}} u_{j}^{*}\right)^{n-1} \\
& \leq \int_{\Omega}\left(-g\left(\cdot, w_{j}\right)\right) d d^{\mathrm{c}} h \wedge\left(d d^{\mathrm{c}} u_{j}^{*}\right)^{n-1} \\
& =\int_{\Omega}(-h) d d^{\mathrm{c}} g\left(\cdot, w_{j}\right) \wedge\left(d d^{\mathrm{c}} u_{j}^{*}\right)^{n-1} \leq \ldots \\
& \leq \int_{\Omega}(-h)\left(d d^{\mathrm{c}} g\left(\cdot, w_{j}\right)\right)^{n}=-(2 \pi)^{n} h\left(w_{j}\right) .
\end{aligned}
$$

The integration by parts is justified since all the functions involved are bounded near $\partial \Omega$. Since $h$ is an exhaustion function, $h\left(w_{j}\right) \rightarrow 0$ as $j \rightarrow \infty$. This shows that the measures $\left(d d^{c} u_{j}^{*}\right)^{n}$ tend weakly to 0 . However, $u_{j}^{*}$ is a decreasing sequence of negative plurisubharmonic functions. By continuity of the Monge-Ampère operator on such sequences, $u=\lim _{j} u_{j}^{*}$ is a maximal plurisubharmonic function that is equal to zero on $\partial \Omega$. Hence $u \equiv 0$. Since $u_{j}^{*}$ is decreasing, each $u_{j}^{*}$ must in fact vanish identically on $\Omega$.

Thus, for each $j$, there is a pluripolar set $E_{j}$ such that $u_{j} \equiv 0$ on $\Omega \backslash E_{j}$. Set $E=E_{1}$. The set $E$ has the required properties for the following reason: The sets $E_{j}$ form an increasing sequence. However, if $z \in E_{j} \backslash E$, then $g\left(z, w_{k}\right)=0$ for some $1 \leq k<j$, which is clearly impossible, hence $E_{j}=E_{1}$ for every $j$.

REMARK. If the set $E$ is empty for every sequence tending to $w_{0}$, then $w_{0}$ has Property $\left(\mathrm{P}_{0}\right)$. This can be seen from passing to suitable subsequences.

One drawback of this result is that the exceptional set may depend on the sequence $\left\{w_{n}\right\}$. At the expense of a weaker conclusion, we can find a universal exceptional set. More precisely:

Corollary 4.8. Let $\Omega$ be a bounded, hyperconvex domain in $\mathbb{C}^{N}$, and let $w_{0} \in \partial \Omega$. Then there exists a pluripolar set $\widetilde{E} \subset \Omega$ such that

$$
\varlimsup_{w \rightarrow w_{0}} g(z, w)=0 \quad \text { for every } z \in \Omega \backslash \widetilde{E} .
$$


Pr o of. Let $\mathcal{S}$ denote the set of all sequences in $\Omega$ tending to $w_{0}$. Take

$$
\widetilde{E}=\bigcap_{s \in \mathcal{S}} E_{s}
$$

where $E_{s}$ denotes the exceptional set corresponding to the sequence $s$.

5. A note on the multipolar Green function. Since the complex Monge-Ampère operator is non-linear, it makes sense to consider pluricomplex Green functions with multiple poles. (The same construction for the Laplace operator would only yield the sum of the corresponding one-polar Green functions.) More precisely, let

$$
A=\left\{\left(w_{1}, \nu_{1}\right), \ldots,\left(w_{m}, \nu_{m}\right)\right\}
$$

be a finite subset of $\Omega \times \mathbb{R}_{+}$. Lelong [17] introduced the multipolar Green function with poles in $A$ as

$$
g(z, A)=\sup \left\{u(z): u \in \mathcal{L}_{A}\right\},
$$

where $\mathcal{L}_{A}$ denotes the family of negative plurisubharmonic functions on $\Omega$, having a logarithmic pole of weight $\nu_{j}$ at each $w_{j} \in A$.

As with the (unipolar) Green function, the multipolar Green function for $\Omega$ tends to 0 as $z$ tends to any boundary point if and only if $\Omega$ is hyperconvex. With the help of the following estimates, we can connect the boundary behaviour of the multipolar Green function with the boundary behaviour of the one-pole Green function. We have

$$
\sum_{A^{\prime} \in \mathcal{P}} g\left(z, A^{\prime}\right) \leq g(z, A) \leq \min _{A^{\prime} \in \mathcal{P}} g\left(z, A^{\prime}\right),
$$

where $\mathcal{P}$ is any partition of $A$. These estimates follow immediately from the fact that the sum in the leftmost term is a member of the defining family for $g(\cdot, A)$, and that $g(\cdot, A)$ is a member of the defining family for each function in the rightmost term. In summary, we have the following theorems:

Theorem 5.1. Let $\Omega$ be a domain in $\mathbb{C}^{N}$. Fix $z \in \Omega$ and let $A=$ $\left\{\left(w_{j}, \nu_{j}\right)\right\}$ be a finite subset of $\Omega \times \mathbb{R}_{+}$. If $w_{0} \in \partial \Omega$ has Property $\left(\mathrm{P}_{0}\right)$, then for every sequence $\left\{\omega_{n}\right\}_{n=1}^{\infty}$ such that $\omega_{n} \rightarrow w_{0}$ and every $\nu>0$, we have

$$
g\left(z, A \cup\left\{\left(\omega_{n}, \nu\right)\right\}\right) \rightarrow g(z, A)
$$

as $n \rightarrow \infty$.

Proof. The assumptions imply that $g\left(z, \omega_{n}\right) \rightarrow 0$ as $n \rightarrow \infty$. Hence, by $(5.1)$,

$$
g(z, A)+\nu g\left(z, \omega_{n}\right) \leq g\left(z, A \cup\left\{\left(\omega_{n}, \nu\right)\right\}\right) \leq \min \left\{g(z, A), \nu g\left(z, \omega_{n}\right)\right\} .
$$

Letting $n \rightarrow \infty$, we see that $g\left(z, A \cup\left\{\left(\omega_{n}, \nu\right)\right\}\right) \rightarrow g(z, A)$. 
Theorem 5.2. Let $\Omega$ be a domain in $\mathbb{C}^{N}$. Let $z \in \Omega$ and $\left\{w_{1}, \ldots, w_{m}\right\}$ be a finite subset of $\partial \Omega$. Suppose that $w_{j}$ has Property $\left(\mathrm{P}_{0}\right)$ for every $1 \leq$ $j \leq m$. Take a sequence $\left\{\left(w_{1}^{(n)}, \ldots, w_{m}^{(n)}\right)\right\}_{n=1}^{\infty}$ of m-tuples of points in $\Omega$ such that $w_{j}^{(n)} \rightarrow w_{j}$ as $n \rightarrow \infty$ for every $1 \leq j \leq m$. Then for every $\left(\nu_{1}, \ldots, \nu_{m}\right) \in \mathbb{R}_{+}^{m}$,

$$
g\left(z,\left\{\left(w_{1}^{(n)}, \nu_{1}\right), \ldots,\left(w_{m}^{(n)}, \nu_{m}\right)\right\}\right) \rightarrow 0
$$

as $n \rightarrow \infty$.

Proof. The assumptions imply that, for every $j, g\left(z, w_{j}^{(n)}\right) \rightarrow 0$ as $n \rightarrow \infty$. Hence, by (5.1),

$$
\sum_{j} \nu_{j} g\left(z, w_{j}^{(n)}\right) \leq g\left(z,\left\{\left(w_{1}^{(n)}, \nu_{1}\right), \ldots,\left(w_{m}^{(n)}, \nu_{m}\right)\right\}\right) \leq \min _{j}\left\{\nu_{j} g\left(z, w_{j}^{(n)}\right)\right\} .
$$

Letting $n \rightarrow \infty$, we see that $g\left(z,\left\{\left(w_{1}^{(n)}, \nu_{1}\right), \ldots,\left(w_{m}^{(n)}, \nu_{m}\right)\right\}\right) \rightarrow 0$.

\section{References}

[1] A. Aytuna, On Stein manifolds $M$ for which $\mathcal{O}(M)$ is isomorphic to $\mathcal{O}\left(\Delta^{n}\right)$ as Fréchet spaces, Manuscripta Math. 62 (1988), 297-316.

[2] E. Bedford and J. E. Fornæss, A construction of peak functions on weakly pseudoconvex domains, Ann. of Math. 107 (1978), 555-568.

[3] Z. Błocki, The complex Monge-Ampère operator in hyperconvex domains, Ann. Scuola Norm. Sup. Pisa Cl. Sci. (4) 23 (1996), 721-747.

[4] M. Carlehed, Comparison of the pluricomplex and the classical Green functions, Michigan Math. J. 45 (1998), 399-407.

[5] U. Cegrell, Capacities in Complex Analysis, Aspects Math. E14, Vieweg, 1988.

[6] D. Coman, Remarks on the pluricomplex Green function, preprint, 1996.

[7] K. Diederich and G. Herbort, Pseudoconvex domains of semiregular type, in: Contributions to Complex Analysis and Analytic Geometry, H. Skoda and J.-M. Trépreau (eds.), Aspects Math. E26, Vieweg, 1994, 127-161.

[8] A. Edigarian, On definitions of the pluricomplex Green function, Ann. Polon. Math. 67 (1997), 233-246.

[9] J. E. Fornæss and J. Wiegerinck, Approximation of plurisubharmonic functions, Ark. Mat. 27 (1989), 257-272.

[10] T. Gamelin, Uniform Algebras and Jensen Measures, London Math. Soc. Lecture Note Ser. 32, Cambridge Univ. Press, 1978.

[11] M. Hervé, Lindelöf's principle in infinite dimensions, in: Proc. on Infinite Dimensional Holomorphy (Berlin), T. L. Hayden and T. J. Suffridge (eds.), Lecture Notes in Math. 364, Springer, 1974, 41-57.

[12] M. Jarnicki and P. Pflug, Invariant Distances and Metrics in Complex Analysis, Walter de Gruyter, 1993.

[13] N. Kerzman et J.-P. Rosay, Fonctions plurisousharmoniques d'exhaustion bornées et domaines taut, Math. Ann. 257 (1981), 171-184.

[14] M. Klimek, Pluripotential Theory, London Math. Soc. Monographs (N.S.) 6, Oxford Univ. Press, 1991. 
[15] S. G. Krantz, Function Theory of Several Complex Variables, 2nd ed., Wadsworth \& Brooks/Cole, 1992.

[16] F. Lárusson and R. Sigurdsson, Plurisubharmonic functions and analytic discs on manifolds, J. Reine Angew. Math. 501 (1998), 1-39.

[17] P. Lelong, Fonction de Green pluricomplexe et lemme de Schwarz dans les espaces de Banach, J. Math. Pures Appl. 68 (1989), 319-347.

[18] L. Lempert, La métrique de Kobayashi et la représentation des domaines sur la boule, Bull. Soc. Math. France 109 (1981), 427-474.

[19] - Intrinsic distances and holomorphic retracts, in: Complex Analysis and Applications '81, Bulgar. Acad. Sci., Sophia, 1984, 341-364.

[20] E. A. Poletsky, Holomorphic currents, Indiana Univ. Math. J. 42 (1993), 85-144.

[21] E. A. Poletsky and B. V. Shabat, Invariant metrics, in: Several Complex Variables III, G. M. Khenkin (ed.), Encyclopaedia Math. Sci., 9, Springer, 1989, 63-111.

[22] H. W u, Normal families of holomorphic mappings, Acta Math. 119 (1967), 193-233.

[23] J. Yu, Peak functions on weakly pseudoconvex domains, Indiana Univ. Math. J. 43 (1994), 1271-1295.

[24] W. Zwonek, On Carathéodory completeness of pseudoconvex Reinhardt domains, preprint, 1998.

Mid Sweden University

S-831 25 Östersund, Sweden

Department of Mathematics

E-mail: Magnus.Carlehed@ter.mh.se

Umeå University

S-901 87 Umeå, Sweden

E-mail: Urban.Cegrell@math.umu.se

Frank.Wikstrom@math.umu.se 